\section{Memorizing the floral ABC}

Gerd Jürgens

\section{Homeotic genes control the development of cell groups - from body segments in the fruitfly Drosophila to floral organs. The cloning of the CURL Y LEAF gene in Arabidopsis reveals similar regulation of homeotic genes in this plant as in Drosophila.}

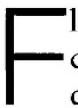

lowers and insects may have more in common than meets the eye. Both are composed of repeated units - whorls of floral organs, or body segments - which owe their specific identities to homeotic genes. Although they are unrelated by DNA sequence, the fly and flower genes have essentially the same biological effects: if they are inactivated or expressed ectopically (in the wrong place), normal structures are formed at the wrong positions - legs where antennae should be or petals in place of stamens. These homeotic transformations (making a structure look like some other structure elsewhere in the body) gave the group of developmental control genes their generic name. The similarities between the two systems have now been taken one step further by Goodrich and colleagues ${ }^{1}$, who report on page 44 of this issue that $C U R L Y L E A F$, a negative regulator of an Arabidopsisfloral homeotic gene, seems to be related to a Polycomb-group repressor of Drosophila homeotic genes.

Flowers have only four types of organs, which are arranged in concentric whorls (Fig. 1): leaf-like sepals in the peripheral whorl 1 , showy petals in whorl 2 , stamens or male reproductive organs in whorl 3 , and carpels or female reproductive organs in the central whorl 4. Homeotic transformations of organs (for example, the conversion of sepals into carpels) in two distantly related plant species, Arabidopsis and Antirrhinum ${ }^{2}$, indicated that simple genetic rules might underlie the construction of the flower. Indeed, these observations led to an elegant unifying concept, the $\mathrm{ABC}$ model ${ }^{3}$, which stipulates that three classes of homeotic gene activities (A, B and C) are sufficient to generate the four types of floral organs in a combinatorial fashion - class A acts in whorls 1 and 2 , class $B$ in whorls 2 and 3 , and class $C$ in whorls 3 and 4 . So class A genes alone make sepals, $A$ and $B$ together make petals, B and C stamens, and class $\mathrm{C}$ genes alone make carpels. To account for the transformation of sepals into carpels and vice versa, it was proposed that the class $\mathrm{A}$ and class $\mathrm{C}$ genes mutually inhibit each other.

The ABC model not only correctly predicted the phenotypes of double and triple mutants, but it also withstood more sophisticated tests. For example, ectopic expression

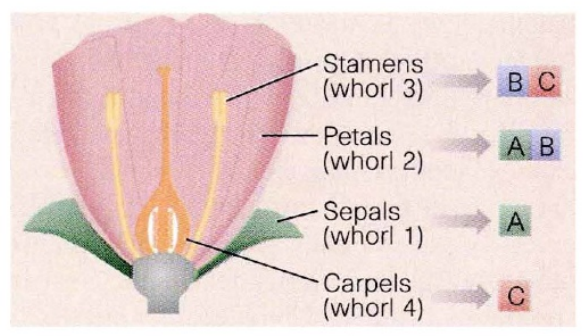

Figure 1 The activities of floral homeotic genes determine the identity of floral organs. According to the $\mathrm{ABC}$ model, class $\mathrm{A}$ genes encode sepals (whorl 1); class A and B genes together make petals (whorl 2); class $B$ and $C$ genes are required to produce stamens (whorl 3); and class $C$ genes alone encode carpels (whorl 4). The class $A$ and class $C$ genes mutually inhibit one another. Goodrich et al.' describe a gene, CURLY LEAF, which seems to repress the expression of a class $\mathrm{C}$ gene in Arabidopsis outside its normal domain.

of class B genes in the absence of A-gene function transformed all floral organs into stamens ${ }^{4}$, as predicted from the model. It is now known that floral homeotic genes encode transcriptional regulators, all of which, with one exception, contain a MADS box (a conserved DNA-binding domain). The genes are transcribed only in those whorls that are affected by mutation in the genes themselves (again with one exception). Transcription starts early in floral-organ development, and continues during the period of growth until the cells differentiate.

How does the CURLY LEAF (CLF) gene fit into this scheme? As the name suggests, clf mutations lead to leaf curling, which can also be caused by ectopic expression of the class $C$ gene AGAMOUS $(A G)^{5}$, or the class B genes ${ }^{4}$ APETALA3 (AP3) and PISTILLATA together. Goodrich et al. ${ }^{1}$ have found that in $c l f$ mutants, $A G$, and to a lesser extent $A P 3$, is indeed expressed in leaves, indicating that the normal function of $C L F$ is to repress $A G$. The outer whorls of clf flowers are also abnormal, and $A G$ transcripts accumulate in petals during the later stages of flower development (although the initial expression of $A G$ is normal).

What, then, is the developmental significance of the CLF gene? A comparison with Drosophila development might give a clue.
Here, homeotic selector genes are activated in the primordia that give rise to specific segments, in response to transient signals from segmentation genes. The initial pattern of active and inactive genes is subsequently maintained through many rounds of cell division $^{6}$. The maintenance (or memory) system involves Polycomb-group genes, which are involved in continuous repression. These genes were named after the dominant mutant-phenotype of the founder gene of the group - male Drosophila not only have sex combs on their forelegs, but also on their midlegs and hindlegs. Most of the Polycombgroup genes that have been analysed so far encode structurally diverse chromosomal proteins ${ }^{7}$. Mutations in Polycomb-group genes result in ectopic expression of homeotic selector genes, for example, the Bithoraxcluster genes; however, this only occurs at a later stage of embryogenesis.

The functional similarities indicate that the $C L F$ gene and the Polycomb-group genes may have comparable functions in maintaining the inactive state of homeotic genes. The startling observation made by Goodrich et $a .^{1}{ }^{1}$ is that the deduced CLF protein sequence shares extensive stretches of amino acids - such as the functionally uncharacterized SET domain, and several cysteinerich domains - with the chromosomal protein Enhancer of zeste ${ }^{8}$, which is a Drosophila Polycomb-group member.

The new results raise several questions. Is $C L F$ just the first representative of a group of functionally, but not necessarily structurally, related maintenance genes in plants? If this were the case, would double mutants show a more complete transformation of homeotic units, as Polycomb-group double mutants do in Drosophila? And are other aspects of plant development affected? The clf mutant could also be used to address a long-standing question in plant development. Plant cells have long been viewed as being flexible in their developmental fates, and subject to continuous signals from their surroundings. In contrast, stable expression of homeotic gene activities in Drosophila confers identity in a cell-autonomous fashion from early in development. These two views are not necessarily mutually exclusive, and generating clones of mutant clf cells might be a means of assaying for autonomous cell-fate decisions in plant development.

Gerd Jürgens is in the Lehrstuhl für Entwicklungsgenetik, University of Tübingen, Spemannstrasse 37-39, D-72076 Tübingen, Germany.

\footnotetext{
1. Goodrich, J. et al. Nature 386, 44-51 (1997).

2. Coen, E. S. \& Meyerowitz, E. M. Nature 353, 31-37 (1991).

3. Weigel, D. \& Meyerowitz, E. M. Cell 78, 203-209 (1994).

4. Krizek, B. A. \& Meyerowitz, E. M. Development 122, 11-22 (1996).

5. Mizukami, Y. \& Ma, H. Cell 71, 119-131 (1992).

6. Lawrence, P. A. \& Morata, G. Cell 78, 181-189 (1994).

7. Kennison, J.A. Annu. Rev. Genet. 29, 289-303 (1995).

8. Carrington, E. A. \& Jones, R. S. Development 122, 4073-4083 (1996).
} 